\title{
Erratum to: The system modulating ROS content in germinating seeds of two Brazilian savanna tree species exposed to As and Zn
}

\author{
M. P. Gomes • M. M. L. C. Carneiro • \\ C. O. G. Nogueira • A. M. Soares · Q. S. Garcia
}

Published online: 9 February 2013

(C) Franciszek Górski Institute of Plant Physiology, Polish Academy of Sciences, Kraków 2013

\section{Erratum to: Acta Physiol Plant}

DOI 10.1007/s11738-012-1140-6

Unfortunately, the original publication of Fig. 2 contained errors. The corrected Fig. 2 is reproduced on the following page.

The online version of the original article can be found under doi:10.1007/s11738-012-1140-6.

M. P. Gomes ( $\square)$

Institut des Sciences de l'environnement, Université du Québec à

Montréal, Succ. Centre-Ville, C.P. 8888, Montreal

QC H3C 3P8, Canada

e-mail: marcelopgom@yahoo.com.br

M. P. Gomes · M. M. L. C. Carneiro .

C. O. G. Nogueira - A. M. Soares

Departamento de Biologia, Universidade Federal de Lavras

(UFLA), Campus UFLA, C.P. 3037, Lavras

MG 37200-000, Brazil

Q. S. Garcia

Departamento de Botânica, Instituto de Ciências Biológicas,

Universidade Federal de Minas Gerais (UFMG), Avenida

Antonio Carlos 6627 Pampulha, C.P. 486, Belo Horizonte

MG 31270-970, Brazil

e-mail: queilagarcia@gmail.com 


\section{Anadenanthera peregrina}
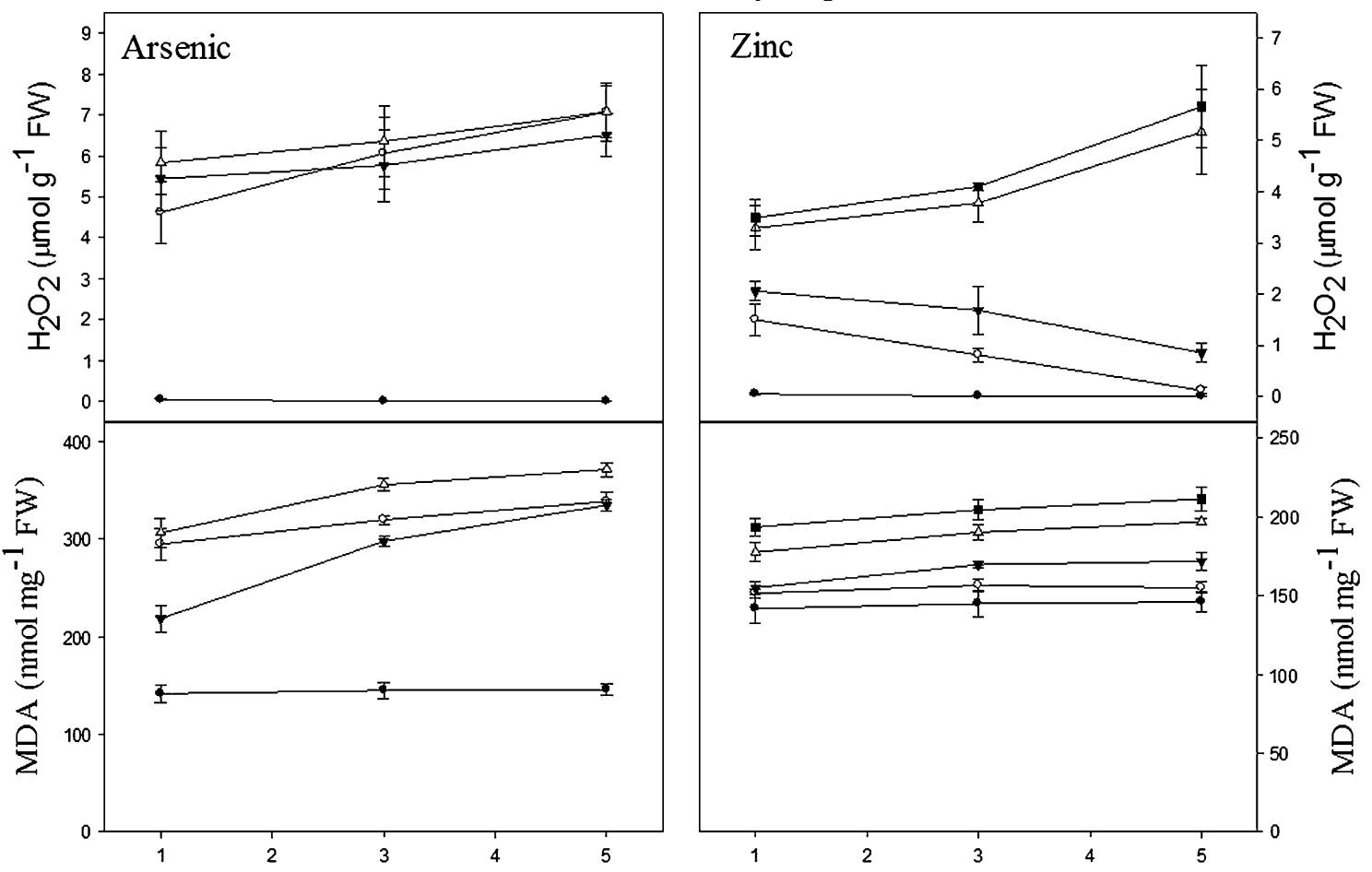

Myracrodruon urundeuva
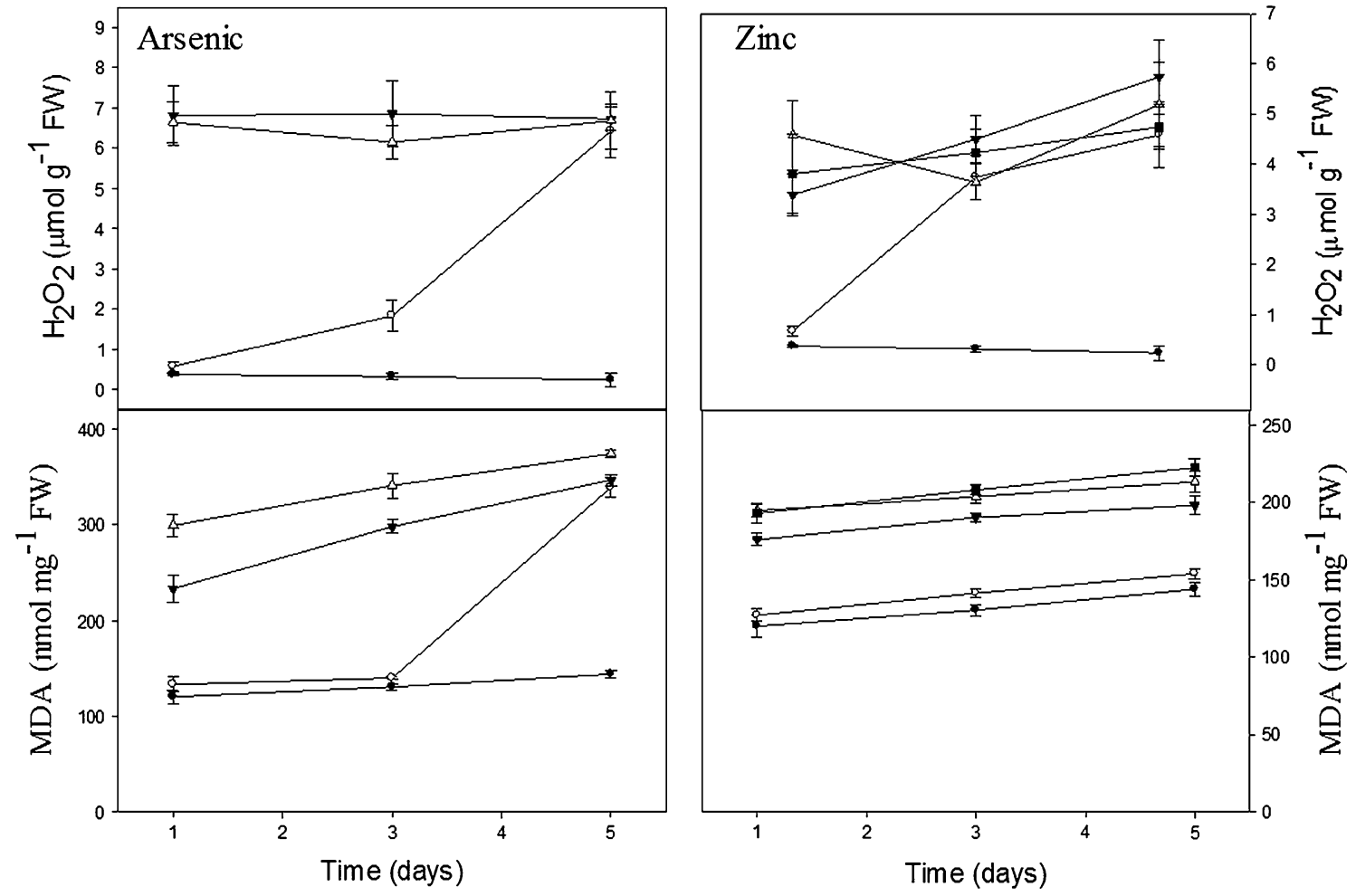

Fig. 2 Time-course of $\mathrm{H}_{2} \mathrm{O}_{2}$ concentration and lipid peroxidation (MDA) in Anadenanthera peregrina and Myracrodruon urundeuva seeds exposed to increasing arsenic $\left(0,10,50\right.$, and $\left.100 \mathrm{mg} \mathrm{L}^{-1}\right)$ and zinc $\left(0,80,120\right.$, and $\left.200 \mathrm{mg} \mathrm{L}^{-1}\right)$ doses. Values are means $\pm \mathrm{SE}$ of five replicates. The concentrations of added As were 0 (filled circle),

10 (open circle), 50 (filled inverted triangle), and 100 (open triangle) $\mathrm{mg} \mathrm{L}{ }^{-1}$. The levels of added $\mathrm{Zn}$ were 0 (filled circle), 50 (open circle), 80 (filled inverted triangle), 120 (open triangle), and 200 (filled square) $\mathrm{mg} \mathrm{L}^{-1}$ 